\title{
New analytic solutions for wave propagation in flexible, tapered vessels with reference to mammalian arteries
}

\author{
George Papadakis $\dagger$ \\ Department of Aeronautics, Imperial College London, Exhibition Road, London SW7 2AZ, UK
}

(Received 18 December 2010; revised 21 September 2011; accepted 26 September 2011; first published online 17 November 2011)

Novel, closed-form, analytic solutions for the pressure and velocity fields are derived for the linear problem of wave propagation inside a tapered flexible vessel of conical shape. It is shown that pressure and velocity can be written in terms of Bessel functions of orders $1 / 3$ and $4 / 3$ respectively. An expression is also derived that quantifies the effect of the cone angle on the wave propagation velocity. The analytic solutions are general and valid for tube variations at any length scale in relation to the wavelength of the wave. In other words, the requirement that the changes in vessel properties with distance should take place over a length scale large compared to the wavelength of the wave, is not employed or needed. This is the basic condition for the application of WKB theory to tapered vessels. However, this condition is not satisfied in pressure pulses propagating in mammalian arteries. The general expressions derived in this paper are directly applicable to the cardiovascular system of mammals. It is further shown that the presented solution naturally tends to the asymptotic WKB solution when the assumptions of the theory are applied to the general expressions. An explicit formula is provided for the time-averaged energy flux of the wave that shows clearly the effect of the continuous reflection of the wave from the vessel wall. Viscous effects are incorporated by coupling the derived analytic solution with the radial velocity profile of Womersley. The results are compared with full nonlinear fluid-structure interaction simulations and very good agreement is found (maximum differences are $\sim 1 \%$ and $1.6 \%$ for area-averaged pressure and velocity respectively, and $4-6 \%$ for local velocity values).

Key words: blood flow, flow-vessel interactions

\section{Introduction}

The theory of pulse wave propagation in arteries has a long and rich history. In the nineteenth century Moens (1877) published experimental work on wave propagation in arteries and Korteweg (1878) derived an equation for the wave speed in thin elastic vessels, now known as the Moens-Korteweg equation. Witzig (1914) was the first to show the effects of fluid viscosity on propagation characteristics, impedance and velocity profiles. Significant developments of the theory took place in the 1950 s and 1960s: Womersley (1955, 1957), Atabek \& Lew (1961) and Atabek (1968), among

$†$ Email address for correspondence: g.papadakis@ic.ac.uk 
many others). A survey of the work in the area until the late 1960s is provided by Cox (1969). More recent reviews can be found in Pedley $(2000,2003)$ and Van de Vosse \& Stergiopoulos (2011).

The nonlinear equations for one-dimensional wave propagation in flexible vessels can be derived from first principles (mass conservation and momentum balance). The equations are closed with a constitutive expression for the effect of pressure on the size of the cross-sectional area of the vessel (Hughes \& Lubliner 1973; Sherwin et al. 2003; Bessems, Rutten \& van de Vosse 2007). The effect of nonlinearity has been examined and was found to have only a secondary contribution under physiological conditions. Only a slight steepening of the wave form in the aorta can be observed (Pedley 2003; Sherwin et al. 2003; Matthys et al. 2007). On the other hand, the effect of wall visco-elasticity on pulse propagation can be more significant. Bessems et al. (2008) performed experiments and simulations of wave propagation in polyurethane vessels and found that a linear elastic material law underestimates the damping of the system, while accounting for the visco-elastic wall properties increases the damping and significantly improves the predictions of pressure and flow rate wave forms. More recently Alastruey et al. (2011) published a new assessment of the one-dimensional visco-elastic equations in a distributed silicone network and reached the same conclusions as did Bessems et al. (2008) for a single tube. In vivo, the effect of adding visco-elasticity to the one-dimensional formulation has been examined by Reymond et al. (2009). They found that energy losses and damping effects due to wall visco-elasticity become more significant in the peripheral branches. Steele et al. (2011) developed a general framework to couple a one-dimensional model with a large number of visco-elastic models.

Analytical solutions in the frequency domain for the one- or two-dimensional models can be found only for straight vessels. In practice, however, arteries are tapering vessels (Steele, Olufsen \& Taylor 2007). The rate of tapering appears to be quite variable from animal to animal. In dogs for example, the area fits an exponential form $A=A_{o}\left(1-\mathrm{e}^{-\beta x / r_{o}}\right)$, where $A_{o}$ and $r_{o}$ are the area and radius at a given location, $x$ is the distance from that location and $\beta$ is a dimensionless parameter in the range 0.02-0.05 (Pedley 1980; Fung 1996). Linearization of this equation shows the tapering angle is small, between $0.6-1.5^{\circ}$.

In other to analyse theoretically the wave propagation in tapered vessels, the tapering is considered to be mild and the vessel is approximated by a series of straight vessels of varying radius (Lighthill 1975). The energy of the reflected wave is proportional to the square of the reflection coefficient, $R^{2}$, and if $R \ll 1$ it can be neglected. For tapering vessels, $R$ is proportional to the difference in the crosssectional area of the two successive segments divided by the sum of the areas, and is obviously very small if the tapering is mild ( $R^{2}$ is another order of magnitude smaller). Therefore very little energy is reflected as the wave travels along a slowly varying vessel and the wave is analysed assuming that all energy is transmitted (Fung 1996).

The previous argument can be put in solid mathematical formulation using WKB theory, named after Wentzel, Kramers and Brillouin who popularized the method; see Holmes (1998) and Bender \& Orszag (1999) for an explanation of the theory. The basic assumption of the theory is that the rate of change of wall properties with distance along the tube is slow, i.e. takes place in a length scale which is large with respect to the wavelength of the wave. This allows the introduction of a new, slow variable and it is assumed that the characteristic impedance and wave speed are functions of this variable. This assumption allows the derivation of a simple equation that shows that the pressure pulse and the flow rate vary with rate $Y^{-1 / 2}$ and $Y^{1 / 2}$ 
respectively, where $Y$ is the characteristic admittance (defined as $Y=A /(\rho \lambda), A$ being the cross-sectional area, $\rho$ the fluid density and $\lambda$ the wave speed) that decreases along the vessel. According to the theory, all the energy of the incident wave is transmitted. For details on the derivations, see Pedley (1980).

This theory is very attractive and elegant, but unfortunately cannot be applied directly to the pressure pulse propagation in the cardiovascular tree of mammals. For example the wave length of the fundamental wave (with frequency equal to $2 \mathrm{~Hz}$ in dogs) is several metres while the length of the longest vessel, the aorta, is of the order of $0.5 \mathrm{~m}$ (Pedley 1980). This means that the area of the aorta has reduced significantly along a distance which is a fraction of the wavelength.

It is exactly at this point that the present paper aims to contribute. We derive general analytic solutions in closed form that do not make the aforementioned assumption and are therefore valid for the conditions encountered in the cardiovascular tree of mammals. In order to validate the theory we compare the results with full nonlinear fluid-structure interaction simulations.

Transmission line theory has also been applied to the modelling of wave propagation in flexible vessels. For tapering vessels in particular, the non-uniform transmission theory has been employed by some investigators (Einav, Aharoni \& Manoach 1998; Myers \& Capper 2004). A nonlinear Riccati equation for the input impedance as a function of the distance along the vessels is solved by Myers \& Capper (2004). The analytic solution for the input impedance can be written in terms of modified Bessel functions of complex argument and order. The orders of the Bessel functions are combinations of the complex numbers representing the characteristic impedance and propagation (an exponential variation is assumed for both with respect to the distance along the vessel).

In this paper we derive a general solution of the governing one-dimensional wave propagation equations written in velocity/pressure variables. The paper is organized as follows. Section 2 presents the mathematical formulation of the one-dimensional wave propagation model, while the eigen-structure of the hyperbolic system of equations is examined in $\S 3$. This analysis results in an expression for the wave propagation velocity and provides explicit formulas for the characteristic (Riemann) variables and directions. The analytic solution of the system in the frequency domain and the asymptotic result for slow varying wall properties is given in $\S 4$. Viscous effects are incorporated into the solution at $\S 5$. Comparison with nonlinear fluid-structure interaction simulations is given in $\S 6$, while $\S 7$ summarizes the main findings of the paper.

\section{Mathematical formulation}

\subsection{Area-averaged equations}

We assume a flexible conical vessel of length $L$ and angle $a$, as shown in figure 1 . The variable ' $s$ ' is used to denote the distance from the tip of the cone (denoted by $O$ ) and the variable ' $r$ ' is reserved for the local radius of the cone (measured from the cone axis). The relation between the two variables is $r=s \sin a$. Distance $s$ varies from $s_{2}$ to $s_{1}$, angle $\theta$ from 0 to $a$ and $\phi$ (the circumferential angle around the cone axis) from 0 to $2 \pi$. Note that $s$ is opposite to $x$, a variable used to measure the axial distance along the vessel. The inlet of the vessel is at $x=0$ and the outlet at $x=L$. The relation between $x$ and $s$ is $x=\left(s_{1}-s\right) \cos a$.

The Navier-Stokes and continuity equations are written in a spherical coordinate system $(s, \phi, \theta)$ with centre at $O$ as shown in figure 1 . This is the most convenient 


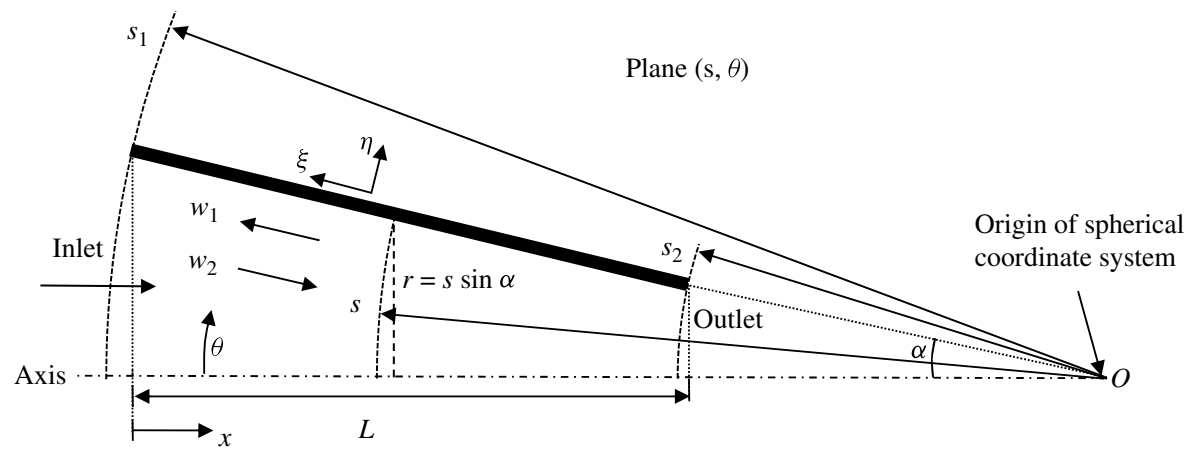

FIGURE 1. Sketch of the geometry under consideration in plane $(s, \theta)$ and definition of basic variables.

coordinate system for the problem examined because the equations take a simpler form. Moreover, the vessel wall corresponds to a constant value of one of the coordinates $(\theta=a)$. As will be shown later, this makes the implementation of wall deformation into the equations straightforward.

Assuming inviscid, incompressible and axisymmetric flow (i.e. $u_{\varphi}=0$ and all derivatives with respect to $\phi$ are equal to zero), the equations in this coordinate system are (see Warsi 1999)

$$
\begin{gathered}
\frac{1}{s^{2}} \frac{\partial}{\partial s}\left(s^{2} u_{s}\right)+\frac{1}{s \sin \theta} \frac{\partial}{\partial \theta}\left(u_{\theta} \sin \theta\right)=0, \\
\frac{\partial u_{s}}{\partial t}+u_{s} \frac{\partial u_{s}}{\partial s}+\frac{u_{\theta}}{s} \frac{\partial u_{s}}{\partial \theta}-\frac{u_{\theta}^{2}}{s}=-\frac{1}{\rho} \frac{\partial p}{\partial s}, \\
\frac{\partial u_{\theta}}{\partial t}+u_{s} \frac{\partial u_{\theta}}{\partial s}+\frac{u_{\theta}}{s} \frac{\partial u_{\theta}}{\partial \theta}+\frac{u_{s} u_{\theta}}{s}=-\frac{1}{\rho} \frac{\partial p}{s \partial \theta} .
\end{gathered}
$$

In this set, $u_{s}, u_{\theta}$ denote the fluid velocities in the $s$ and $\theta$ directions respectively and $t$ the time. Equation (2.1a) represents the continuity equation, $(2.1 b)$ the momentum equation in the $s$ direction, and $(2.1 c)$ the momentum equation in the $\theta$ direction. The assumptions of incompressibility and axially symmetric flow are valid for the purposes of this paper. We assume further that there is no steady (i.e. mean) velocity component; in other words the flow velocities are induced only by the pressure wave, and they are small. This assumption simplifies the resulting system of equations. Under these conditions the equations can be linearized by ignoring the convection terms and take the form

$$
\begin{gathered}
\frac{1}{s^{2}} \frac{\partial}{\partial s}\left(s^{2} u_{s}\right)+\frac{1}{s \sin \theta} \frac{\partial}{\partial \theta}\left(u_{\theta} \sin \theta\right)=0, \\
\frac{\partial u_{s}}{\partial t}=-\frac{1}{\rho} \frac{\partial p}{\partial s}, \\
\frac{\partial u_{\theta}}{\partial t}=-\frac{1}{\rho} \frac{\partial p}{s \partial \theta} .
\end{gathered}
$$

If a steady component, $U_{s t}(s)$, were present, then $(2.2 b)$ and $(2.2 c)$ would contain additional terms. For example the linearization of convection term $u_{s}\left(\partial u_{s} / \partial s\right)$ in $(2.1 b)$ would give the extra terms $U_{s t}\left(\partial u_{s} / \partial s\right)+u_{s}\left(\partial U_{s t} / \partial s\right)$. As mentioned in the Introduction, the linearization of the equations is justified because the nonlinear terms have only 
secondary importance in physiological flow conditions. The next step is to integrate the above set of equations in a surface of constant $s$ (one such surface is shown in figure 1):

$$
\begin{gathered}
\int_{S}\left[\frac{1}{s^{2}} \frac{\partial}{\partial s}\left(s^{2} u_{s}\right)\right] \mathrm{d} A+\int_{S}\left[\frac{1}{s \sin \theta} \frac{\partial}{\partial \theta}\left(u_{\theta} \sin \theta\right)\right] \mathrm{d} A=0, \\
\int_{S} \frac{\partial u_{s}}{\partial t} \mathrm{~d} A=-\frac{1}{\rho} \int_{S} \frac{\partial p}{\partial s} \mathrm{~d} A, \\
\int_{S} \frac{\partial u_{\theta}}{\partial t} \mathrm{~d} A=-\frac{1}{\rho} \int_{S} \frac{1}{s} \frac{\partial p}{\partial \theta} \mathrm{d} A .
\end{gathered}
$$

The surface element $\mathrm{d} A$ in spherical coordinates is $\mathrm{d} A=s^{2} \sin \theta \mathrm{d} \theta \mathrm{d} \varphi$, and since $s$ is constant we can exchange the order of integration and differentiation with respect to $s$, arriving at

$$
\begin{gathered}
\frac{\partial}{\partial s}\left(\int_{0}^{2 \pi} \int_{0}^{a+\delta a} s^{2} u_{s} \sin \theta \mathrm{d} \theta \mathrm{d} \varphi\right)+\int_{0}^{2 \pi} \int_{0}^{a+\delta a} s \frac{\partial}{\partial \theta}\left(u_{\theta} \sin \theta\right) \mathrm{d} \theta \mathrm{d} \varphi=0, \\
\int_{0}^{2 \pi} \int_{0}^{a+\delta a} \frac{\partial u_{s}}{\partial t} s^{2} \sin \theta \mathrm{d} \theta \mathrm{d} \varphi=-\frac{1}{\rho} \frac{\partial}{\partial s} \int_{0}^{2 \pi} \int_{0}^{a+\delta a} p s^{2} \sin \theta \mathrm{d} \theta \mathrm{d} \varphi .
\end{gathered}
$$

The third equation becomes redundant. In (2.4a) and (2.4b), $\delta a$ is a function of $s$ and $t$ and denotes the deviation from $a$ of the local angle in the $\theta$ direction at distance $s$, i.e. the deformed surface is described by the variables $(s, a+\delta a(s, t))$. This set of equations is nonlinear because of the presence of two unknowns in the same term: deviation angle $\delta a$ and velocity $u_{s}$ or $u_{\theta}$ or pressure $p$. Expanding the integrals in $\theta$ direction from 0 to $a$ and from $a$ to $a+\delta a$ in each term, we have

$$
\begin{aligned}
& \frac{\partial}{\partial s} \int_{0}^{2 \pi}\left(\int_{0}^{a} s^{2} u_{s} \sin \theta \mathrm{d} \theta+\underline{\int_{a}^{a+\delta a}} s^{2} u_{s} \sin \theta \mathrm{d} \theta\right) \mathrm{d} \varphi \\
& +\int_{0}^{2 \pi}\left(\int_{0}^{a} s \frac{\partial}{\partial \theta}\left(u_{\theta} \sin \theta\right) \mathrm{d} \theta+\underline{\left.\int_{a}^{a+\delta a} s \frac{\partial}{\partial \theta}\left(u_{\theta} \sin \theta\right) \mathrm{d} \theta\right) \mathrm{d} \varphi=0}\right. \\
& \quad \int_{0}^{2 \pi}\left(\int_{0}^{a} \frac{\partial u_{s}}{\partial t} s^{2} \sin \theta \mathrm{d} \theta+\underline{\left.\int_{a}^{a+\delta a} \frac{\partial u_{s}}{\partial t} s^{2} \sin \theta \mathrm{d} \theta\right) \mathrm{d} \varphi}\right. \\
& \quad=-\frac{1}{\rho} \frac{\partial}{\partial s} \int_{0}^{2 \pi}\left(\int_{0}^{a} p s^{2} \sin \theta \mathrm{d} \theta+\underline{\left.\int_{a}^{a+\delta a} p s^{2} \sin \theta \mathrm{d} \theta\right) \mathrm{d} \varphi}\right.
\end{aligned}
$$

Within every bracket, the underlined term is of second order (integration on a small interval $\delta a$ of small velocity or pressure) and can be ignored with respect to the first term, leading to the linear set

$$
\begin{gathered}
\frac{\partial}{\partial s} \int_{0}^{2 \pi} \int_{0}^{a} s^{2} u_{s} \sin \theta \mathrm{d} \theta \mathrm{d} \varphi+\int_{0}^{2 \pi} \int_{0}^{a} s \frac{\partial}{\partial \theta}\left(u_{\theta} \sin \theta\right) \mathrm{d} \theta \mathrm{d} \varphi=0 \\
\int_{0}^{2 \pi} \int_{0}^{a} \frac{\partial u_{s}}{\partial t} s^{2} \sin \theta \mathrm{d} \theta \mathrm{d} \varphi=-\frac{1}{\rho} \frac{\partial}{\partial s} \int_{0}^{2 \pi} \int_{0}^{a} p s^{2} \sin \theta \mathrm{d} \theta \mathrm{d} \varphi .
\end{gathered}
$$

The second term in $(2.6 a)$ can be integrated analytically:

$$
\frac{\partial}{\partial s}\left(s^{2} \int_{0}^{2 \pi} \int_{0}^{a} u_{s} \sin \theta \mathrm{d} \theta \mathrm{d} \varphi\right)+2 \pi s u_{\theta}(a) \sin a=0,
$$


where the boundary condition $u_{\theta}=0$ at the centreline was used and the velocity $u_{\theta}(a)$ at $\theta=a$ is different from 0 and will be determined from the wall equations. We now introduce the section-averaged velocity and pressure $\bar{u}_{s}(s, t)$ and $\bar{p}(s, t)$ as

$$
\begin{gathered}
\bar{u}_{s}(s, t)=\frac{\int_{0}^{2 \pi} \int_{0}^{a} u_{s} s^{2} \sin \theta \mathrm{d} \theta \mathrm{d} \varphi}{\int_{0}^{2 \pi} \int_{0}^{a} s^{2} \sin \theta \mathrm{d} \theta \mathrm{d} \varphi}=\frac{\int_{0}^{2 \pi} \int_{0}^{a} u_{s} \sin \theta \mathrm{d} \theta \mathrm{d} \varphi}{2 \pi(1-\cos a)}, \\
\bar{p}(s, t)=\frac{\int_{0}^{2 \pi} \int_{0}^{a} p \sin \theta \mathrm{d} \theta \mathrm{d} \varphi}{2 \pi(1-\cos a)} .
\end{gathered}
$$

Using these definitions, equations (2.6) become

$$
\begin{gathered}
\frac{\partial}{\partial s}\left(s^{2} \bar{u}_{s}\right)+s u_{\theta}(a) \frac{\sin a}{1-\cos a}=0, \\
\frac{\partial \bar{u}_{s}}{\partial t}=-\frac{1}{\rho} \frac{\partial \bar{p}}{\partial s} .
\end{gathered}
$$

This set must be complemented with the wall equations that will provide a link between $u_{\theta}(a)$ and the rate of change of $\bar{p}$. The variation of the wall-normal displacement $\eta$ (see figure 1), with respect to the internal pressure $p$ in a conical ring, is given by (A 5) derived in the Appendix. The velocity $u_{\theta}(a)=\mathrm{d} \eta / \mathrm{d} t$, and therefore

$$
u_{\theta}(a)=\frac{\mathrm{d} \eta}{\mathrm{d} t}=\frac{\partial \bar{p}}{\partial t} \frac{\left(1-v^{2}\right) \tan ^{2} a}{E h} s^{2} .
$$

Biological tissue is, to a good approximation, an incompressible material with Poisson ratio $v=0.5$ (Pedley 1980). Equations (2.9) then become

$$
\begin{gathered}
\frac{\partial}{\partial s}\left(s^{2} \bar{u}_{s}\right)+\left(s^{3} \frac{\tan ^{2} a \sin a 0.75}{E h(1-\cos a)}\right) \frac{\partial \bar{p}}{\partial t}=0, \\
\frac{\partial \bar{u}_{s}}{\partial t}=-\frac{1}{\rho} \frac{\partial \bar{p}}{\partial s} .
\end{gathered}
$$

If we group all the geometrical and material parameters into a single variable $f=(0.75 / E h)\left(\tan ^{2} a \sin a /(1-\cos a)\right)$, we have

$$
\begin{array}{r}
\frac{\partial \bar{p}}{\partial t}+\frac{1}{f s} \frac{\partial \bar{u}_{s}}{\partial s}+\frac{2}{f s^{2}} \bar{u}_{s}=0, \\
\frac{\partial \bar{u}_{s}}{\partial t}=-\frac{1}{\rho} \frac{\partial \bar{p}}{\partial s} .
\end{array}
$$

This is the derived system that describes mathematically the variation of pressure and velocity with $s$ and $t$. The eigen-analysis of system (2.12) is presented in the next section. In the following we omit the overbars for the area-averaged velocity and pressure for simplicity.

\section{Eigen-analysis of the pressure-velocity system}

\subsection{Velocities of propagation}

The system of (2.12) can be written in matrix form as

$$
\frac{\partial q}{\partial t}+\boldsymbol{A} \frac{\partial q}{\partial s}=\boldsymbol{B} q,
$$


where

$$
q=\left[\begin{array}{l}
p \\
u_{s}
\end{array}\right], \quad \boldsymbol{A}=\left[\begin{array}{cc}
0 & (f s)^{-1} \\
\rho^{-1} & 0
\end{array}\right] \text { and } \boldsymbol{B}=\left[\begin{array}{cc}
0 & -2\left(f s^{2}\right)^{-1} \\
0 & 0
\end{array}\right] .
$$

Matrix $\boldsymbol{A}$ is diagonalizable with real eigenvalues, so this is a hyperbolic system of equations (LeVeque 2002). The eigenvalues of $\boldsymbol{A}$ represent the velocities of propagation:

$$
\lambda(s)= \pm(\rho f s)^{-1 / 2}= \pm\left(\rho \frac{0.75 s}{E h} \frac{\sin a \tan ^{2} a}{1-\cos a}\right)^{-1 / 2} .
$$

This expression shows the dependence of the wave propagation velocity on distance $s$ and cone angle $a$. It can be written in terms of the local vessel diameter $d=2 r$, where $r$ is measured from the cone axis, as

$$
\lambda(s)= \pm\left(\frac{E h}{0.75 \rho \mathrm{d}}\right)^{1 / 2}\left[\frac{2(1-\cos a)}{\tan ^{2} a}\right]^{1 / 2} .
$$

The first factor represents the well-known Moens-Korteweg equation for cylindrical vessel without longitudinal deformation (plane strain conditions), in which the local diameter of the vessel, $d$, is used. The second term represents a correction factor due to the cone angle. Taylor series expansion of this term gives

$$
\left[\frac{2(1-\cos a)}{\tan ^{2} a}\right]^{1 / 2}=1-\frac{3}{8} a^{2}+\cdots,
$$

which shows that the effect of the angle is of second order $O\left(a^{2}\right)$. For small angles (less than $5^{\circ}$ ), the correction is small.

\subsection{Characteristic variables and directions}

Using the similarity transformation $\boldsymbol{\Lambda}=\boldsymbol{R}^{-1} \boldsymbol{A} \cdot \boldsymbol{R}$, where $\boldsymbol{R}$ is the matrix of eigenvectors $\boldsymbol{R}=\left[\begin{array}{cc}\lambda \rho & -\lambda \rho \\ 1 & 1\end{array}\right], \boldsymbol{A}$ can be diagonalized to the matrix $\boldsymbol{\Lambda}=\left[\begin{array}{cc}\lambda & 0 \\ 0 & -\lambda\end{array}\right]$ (LeVeque 2002). The characteristic variables $w=\left[\begin{array}{l}w_{1} \\ w_{2}\end{array}\right]$ are then defined as $w=\boldsymbol{R}^{-1} q$ or

$$
\begin{gathered}
w_{1}=\frac{1}{2 \lambda \rho}\left(p+\lambda \rho u_{s}\right), \\
w_{2}=\frac{1}{2 \lambda \rho}\left(-p+\lambda \rho u_{s}\right) .
\end{gathered}
$$

Multiplying (3.1) from the left with $\boldsymbol{R}^{-1}$ and writing $\boldsymbol{A}$ as $\boldsymbol{A}=\boldsymbol{R} \boldsymbol{\Lambda} \boldsymbol{R}^{-1}$, we get

$$
\boldsymbol{R}^{-1} \frac{\partial q}{\partial t}+\boldsymbol{\Lambda} \boldsymbol{R}^{-1} \frac{\partial q}{\partial s}=\boldsymbol{R}^{-1} \boldsymbol{B} q \Rightarrow \frac{\partial w}{\partial t}+\boldsymbol{\Lambda} \frac{\partial w}{\partial s}=\left(\boldsymbol{\Lambda} \frac{\partial \boldsymbol{R}^{-1}}{\partial s}+\boldsymbol{R}^{-1} \boldsymbol{B}\right) \boldsymbol{R} w
$$

The left-hand side of the previous equation can be simplified if we define the characteristic directions (or coordinates), $X_{1}, X_{2}$, which can be found be solving the equation

$$
\frac{\mathrm{d} s}{\mathrm{~d} t}= \pm(\rho f s)^{-1 / 2}
$$


Integrating analytically, the characteristic coordinates are found to be

$$
\begin{aligned}
& X_{1}=\frac{2}{3} \sqrt{\rho f} s^{3 / 2}+t, \\
& X_{2}=\frac{2}{3} \sqrt{\rho f} s^{3 / 2}-t .
\end{aligned}
$$

Using these new independent variables, system (3.7) becomes

$$
\begin{aligned}
& \frac{\partial w_{1}}{\partial X_{1}}=g(s) w_{1}+h(s) w_{2}, \\
& \frac{\partial w_{2}}{\partial X_{2}}=h(s) w_{1}+g(s) w_{2},
\end{aligned}
$$

where

$$
g(s)=-\left(\frac{1}{4} \frac{\mathrm{d} \lambda}{\mathrm{d} s}+\frac{1}{2} \frac{\lambda}{s}\right), \quad h(s)=\frac{1}{4} \frac{\mathrm{d} \lambda}{\mathrm{d} s}-\frac{1}{2} \frac{\lambda}{s} .
$$

This is a system of two coupled ordinary differential equations. It can be easily solved numerically with the method of characteristics.

The direction of propagation of $w_{1}$ and $w_{2}$ is shown in figure 1 . Note that the two characteristic variables are coupled through the functions $g(s), h(s)$ and are not constant along the characteristic lines. Even if $w_{1}$ is set to 0 at $s=s_{2}$, the continuous reflection of $w_{2}$ in the wall will immediately create $w_{1}$ waves that will propagate in the opposite direction. The two waves are thus intimately coupled, they propagate together, and cannot be treated independently.

If the cone angle $a \rightarrow 0$, i.e. the cone tends to a cylinder of constant radius $R$, then the velocity of propagation becomes independent of $s$, i.e. $(\mathrm{d} \lambda / \mathrm{d} s) \rightarrow 0$, and also $\lambda / s=(\lambda / R) \sin a \rightarrow 0$ so $g(s), h(s) \rightarrow 0$ and the two waves are constant along $X_{1}$ and $X_{2}$ and propagate independently. Inverting system (3.6) pressure and velocity can be recovered from $w_{1}$ and $w_{2}$ :

$$
\begin{gathered}
p=\lambda \rho\left(w_{1}-w_{2}\right), \\
u_{s}=w_{1}+w_{2} .
\end{gathered}
$$

\section{Analytic solution of the system in the frequency domain}

\subsection{General form of the solution for velocity and pressure}

Let us assume a variation of pressure and velocity of the form $p(s, t)=P(s) \mathrm{e}^{\mathrm{i} \omega t}$ and $u_{s}(s, t)=U(s) \mathrm{e}^{\mathrm{i} \omega t}$ respectively. Substituting into (2.12) we have

$$
\begin{gathered}
\mathrm{i} \omega P+\frac{1}{f s} \frac{\partial U}{\partial s}+\frac{2}{f s^{2}} U=0, \\
\mathrm{i} \omega U=-\frac{1}{\rho} \frac{\mathrm{d} P}{\mathrm{~d} s} .
\end{gathered}
$$

Eliminating $U$, we get the following second-order equation for the complex pressure amplitude $P$ :

$$
s \frac{\mathrm{d}^{2} P}{\mathrm{~d} s^{2}}+2 \frac{\mathrm{d} P}{\mathrm{~d} s}+\left(\rho f \omega^{2}\right) s^{2} P=0 .
$$

Using the transformation $G=P s$, it can be simplified to

$$
\frac{\mathrm{d}^{2} G}{\mathrm{~d} s^{2}}+\left(\rho f \omega^{2}\right) s G=0 .
$$


New analytic solutions for wave propagation in flexible, tapered vessels

The analytic solution of this equation can be written in closed form as (see Råde \& Westergren 1999)

$$
G=\sqrt{s}\left[A \mathrm{~J}_{1 / 3}\left(\frac{2}{3} \sqrt{\rho f} \omega s^{3 / 2}\right)+B \mathrm{Y}_{1 / 3}\left(\frac{2}{3} \sqrt{\rho f} \omega s^{3 / 2}\right)\right],
$$

where $\mathrm{J}_{1 / 3}, \mathrm{Y}_{1 / 3}$ are Bessel functions of order $1 / 3$ and $A, B$ are constants to be determined from boundary conditions. The solution in terms of the original variables $P$ and $s$ is

$$
P(s)=\frac{1}{\sqrt{s}}\left[A \mathrm{~J}_{1 / 3}(z)+B \mathrm{Y}_{1 / 3}(z)\right],
$$

where $z=2 / 3 \sqrt{\rho f} \omega s^{3 / 2}$ denotes the real argument of the Bessel functions. Substituting in $(4.1 b)$, the analytical solution for the amplitude of the velocity is found to be

$$
U(s)=\left(\frac{f}{\rho}\right)^{1 / 2} \frac{1}{\mathrm{i}}\left[A \mathrm{~J}_{4 / 3}(z)+B \mathrm{Y}_{4 / 3}(z)\right],
$$

where $J_{4 / 3}, Y_{4 / 3}$ are Bessel functions of order $4 / 3$. During the derivation of the above expression, the properties of the Bessel functions $\mathrm{dJ}_{1 / 3} / \mathrm{d} z-(1 / 3 z) \mathrm{J}_{1 / 3}=-\mathrm{J}_{4 / 3}$ and $\mathrm{dY}_{1 / 3} / \mathrm{d} z-(1 / 3 z) \mathrm{Y}_{1 / 3}=-\mathrm{Y}_{4 / 3}$ were used. To the best of our knowledge, the solutions (4.5) and (4.6) have not appeared in the literature before. It is interesting to note that the order of the Bessel functions does not depend on the angle of the cone.

Based on the eigen-analysis presented in $\S 3$, we can provide a physical interpretation of the argument $z$ of the Bessel functions. Based on (3.8), the time $\Delta t$ taken by the wave to arrive at position $s$ starting at $s_{0}$ at $t_{0}$ is given by

$$
\int_{s_{0}}^{s}(\rho f s)^{1 / 2} \mathrm{~d} s=\int_{t_{0}}^{t_{0}+\Delta t} \mathrm{~d} t
$$

The integration on the left-hand side can be performed analytically, and gives

$$
\frac{2}{3} \sqrt{\rho f}\left(s^{3 / 2}-s_{0}^{3 / 2}\right)=\Delta t .
$$

Expressing $\Delta t$ in radians (i.e. $\Delta t=\Delta \varphi / \omega$ ), it is immediately seen that $\Delta \varphi=z-z_{0}$. In other words, $z$ is the time the wave takes to reach location $s$ (starting at $s_{0}=z_{0}=0$ ) expressed in radians. Within one period, $z-z_{0}=2 \pi$.

It is interesting to compute the relative change $\Delta \lambda$ and $\Delta Y$ of the wave propagation velocity $\lambda$ and characteristic admittance of the vessel $Y$ (defined as $Y=A / \rho \lambda$ ) at two locations, $s$ and $s_{0}$, separated by the distance travelled by the wave in one period. We have

$$
\begin{gathered}
\Delta \lambda=\frac{\lambda(s)-\lambda\left(s_{0}\right)}{\lambda\left(s_{0}\right)}=\left(\frac{s}{s_{0}}\right)^{-1 / 2}-1=\left(\frac{z}{z_{0}}\right)^{-1 / 3}-1 \\
=\left(1+\frac{2 \pi}{z_{0}}\right)^{-1 / 3}-1, \\
\Delta Y=\frac{A(s) /(\rho \lambda(s))-A\left(s_{0}\right) /\left(\rho \lambda\left(s_{0}\right)\right)}{A\left(s_{0}\right) /\left(\rho \lambda\left(s_{0}\right)\right)}=\left(\frac{s}{s_{0}}\right)^{5 / 2}-1=\left(\frac{z}{z_{0}}\right)^{5 / 3}-1 \\
=\left(1+\frac{2 \pi}{z_{0}}\right)^{5 / 3}-1 .
\end{gathered}
$$

In order to derive the equations above we have used $\lambda(s)=(\rho f s)^{-1 / 2}$ (3.3) and the fact that $z-z_{0}=2 \pi$. We can then see that as $z_{0}$ increases the relative change of the 
vessel properties $\Delta \lambda$ and $\Delta Y$ at one wavelength decrease. Therefore, if we want to examine the behaviour of the solution for very gradual (i.e. very slow) changes of the vessel properties with respect to the wavelength, in other words if we want to apply the condition of WKB theory as explained in the Introduction, we just have to find the asymptotic behaviour of the solution (4.5) and (4.6) for large values of $z$. This observation will be used later in $\S 4.4$.

\subsection{Energy flux}

Using the previous expressions, we can evaluate the energy flux of the wave, $E(s, t)$, defined as the product of the real parts of pressure and flow rate (see Pedley 1980). It is written in two equivalent forms,

$$
E(s, t)=-\operatorname{Re}\left(U(s) \mathrm{e}^{\mathrm{i} \omega t}\right) \operatorname{Re}\left(P(s) \mathrm{e}^{\mathrm{i} \omega t}\right) A(s)
$$

or

$$
E(s, t)=-\lambda \rho\left(w_{1}^{2}-w_{2}^{2}\right) A(s)=\lambda \rho\left(w_{2}^{2}-w_{1}^{2}\right) A(s),
$$

depending on whether velocity/pressure or the characteristic variables are used. The minus (-) sign in (4.10) arises because the $x$ direction is opposite to the $s$ direction (as already mentioned in $\S 2.1)$ and $A(s)$ is the area $A(s)=2 \pi(1-\cos a) s^{2}$. Using the velocity/pressure form we have

$$
E(s, t)=-\frac{1}{4}\left(U^{*}(s) P^{*}(s) \mathrm{e}^{-2 \mathrm{i} \omega t}+U^{*}(s) P(s)+U(s) P^{*}(s)+U(s) P(s) \mathrm{e}^{2 \mathrm{i} \omega t}\right) A(s),(4.1
$$

and the time-averaged value is

$$
\bar{E}(s)=-\frac{1}{4}\left(U^{*}(s) P(s)+U(s) P^{*}(s)\right) A(s) .
$$

In (4.11) and (4.12) the asterisks denote complex conjugates. Substituting (4.5) and (4.6) into (4.12), after some algebra we get

$$
\bar{E}(s)=-\pi(1-\cos a)\left(\frac{f}{\rho}\right)^{1 / 2} s^{3 / 2} \operatorname{Im}\left(A B^{*}\right)\left(\mathrm{J}_{4 / 3}(z) \mathrm{Y}_{1 / 3}(z)-\mathrm{Y}_{4 / 3}(z) \mathrm{J}_{1 / 3}(z)\right),
$$

where $\operatorname{Im}\left(A B^{*}\right)$ is the imaginary part of the product $A B^{*}$ and $B^{*}$ is the complex conjugate of $B$. Using the property of the Bessel functions $\mathrm{J}_{4 / 3}(z) \mathrm{Y}_{1 / 3}(z)-$ $\mathrm{Y}_{4 / 3}(z) \mathrm{J}_{1 / 3}(z)=2 /(\pi z)$, we get the simplified expression

$$
\bar{E}(s)=-\frac{3}{\rho \omega}(1-\cos a) \operatorname{Im}\left(A B^{*}\right) .
$$

Equation (4.14) shows that the time-averaged energy is independent of $s$, as expected, because there is no mechanism to remove energy from the system (the flow is inviscid and the wall is made of linear, elastic material that does not dissipate energy). The presence of both coefficients $A$ and $B$ shows that the energy flux depends on the boundary conditions on either side of the vessel.

\subsection{Solution for specified pressure at inlet and no incoming wave at outlet}

In order to evaluate the coefficients $A$ and $B$ we need two boundary conditions, one at the inlet and one at the outlet. Of course, these depend on the problem under consideration. In the present section we examine the particular case of prescribed pressure at the inlet and no incoming (incident) wave at the outlet, i.e.

$$
\begin{gathered}
s=s_{1}: p\left(s_{1}, t\right)=P_{0} \mathrm{e}^{\mathrm{i} \omega t} \Rightarrow P\left(s_{1}\right)=P_{0}, \\
s=s_{2}: w_{1}\left(s_{2}, t\right)=0 \Rightarrow P\left(s_{2}\right)+\rho \lambda\left(s_{2}\right) U\left(s_{2}\right)=0 .
\end{gathered}
$$


Therefore we have the following two conditions:

$$
\begin{gathered}
P_{0}=\frac{1}{\sqrt{s_{1}}}\left(A \mathrm{~J}_{1 / 3}\left(z_{1}\right)+B \mathrm{Y}_{1 / 3}\left(z_{1}\right)\right), \\
\frac{1}{\sqrt{s_{2}}}\left(A \mathrm{~J}_{1 / 3}\left(z_{2}\right)+B \mathrm{Y}_{1 / 3}\left(z_{2}\right)\right)+\lambda\left(s_{2}\right) \rho\left(\frac{f}{\rho}\right)^{1 / 2} \frac{1}{\mathrm{i}}\left[A \mathrm{~J}_{4 / 3}\left(z_{2}\right)+B \mathrm{Y}_{4 / 3}\left(z_{2}\right)\right]=0 .
\end{gathered}
$$

Solving the linear system, after some algebra we get

$$
\begin{gathered}
P(s)=P_{0}\left(\frac{s_{1}}{s}\right)^{1 / 2} \frac{C \mathrm{~J}_{1 / 3}(z)+\mathrm{Y}_{1 / 3}(z)}{C \mathrm{~J}_{1 / 3}\left(z_{1}\right)+\mathrm{Y}_{1 / 3}\left(z_{1}\right)}, \\
U(s)=P_{0} \frac{1}{\mathrm{i}}\left(\frac{f}{\rho}\right)^{1 / 2} s_{1}^{1 / 2} \frac{C \mathrm{~J}_{4 / 3}(z)+\mathrm{Y}_{4 / 3}(z)}{C \mathrm{~J}_{1 / 3}\left(z_{1}\right)+\mathrm{Y}_{1 / 3}\left(z_{1}\right)}
\end{gathered}
$$

for the velocity and pressure respectively, where $C$ is given by

$$
C=-\frac{\mathrm{iY}_{1 / 3}\left(z_{2}\right)+\mathrm{Y}_{4 / 3}\left(z_{2}\right)}{\mathrm{iJ}_{1 / 3}\left(z_{2}\right)+\mathrm{J}_{4 / 3}\left(z_{2}\right)} .
$$

Substituting expressions (4.17) to (4.12), we get for the energy flux

$$
\bar{E}(s)=-\left|P_{0}\right|^{2} \frac{3}{\rho \omega}(1-\cos a) \frac{\operatorname{Im}(C) s_{1}}{\left|C \mathrm{~J}_{1 / 3}\left(z_{1}\right)+\mathrm{Y}_{1 / 3}\left(z_{1}\right)\right|^{2}},
$$

where $\operatorname{Im}(C)$ is the imaginary part of $C$.

It is interesting to find the limiting value of the energy flux when $s_{2} \rightarrow s_{1}$, i.e. when the vessel has negligible length (thin ring). In such a case, there is no reflection from the wall, and the energy flux is given by

$$
\bar{E}(s)=\left|P_{0}\right|^{2} \pi(1-\cos a)\left(\frac{f}{\rho}\right)^{1 / 2} s_{1}^{5 / 2} .
$$

The importance of this relation will become evident in the next section.

\subsection{Asymptotic solution for large values of argument $z$}

It was mentioned in $\S 4.1$ that the basic assumption of the WKB theory can be reproduced by taking the argument $z$ of the Bessel functions to be very large. It is known (Press et al. 1997) that the Bessel functions of order $1 / 3$ and $4 / 3$ for large values of $z$ can be approximated as

$$
\begin{gathered}
z \gg 1 / 3: \mathrm{J}_{1 / 3}(z) \approx \sqrt{\frac{2}{\pi z}} \cos \left(z-\frac{5 \pi}{12}\right), \quad \mathrm{Y}_{1 / 3}(z) \approx \sqrt{\frac{2}{\pi z}} \sin \left(z-\frac{5 \pi}{12}\right), \\
z \gg 4 / 3: \mathrm{J}_{4 / 3}(z) \approx \sqrt{\frac{2}{\pi z}} \sin \left(z-\frac{5 \pi}{12}\right), \quad \mathrm{Y}_{4 / 3}(z) \approx-\sqrt{\frac{2}{\pi z}} \cos \left(z-\frac{5 \pi}{12}\right) .
\end{gathered}
$$

Substituting (4.21) into (4.17), after some algebra we get

$$
\begin{array}{r}
P(s)=P_{0}\left(\frac{s_{1}}{s}\right)^{5 / 4} \mathrm{e}^{\mathrm{i}\left(z-z_{1}\right)}, \\
U(s)=-P_{0}\left(\frac{f}{\rho}\right)^{1 / 2} s_{1}^{1 / 2}\left(\frac{s_{1}}{s}\right)^{3 / 4} \mathrm{e}^{\mathrm{i}\left(z-z_{1}\right)} .
\end{array}
$$


From (4.22b), the flow rate $Q(s)=-2 \pi(1-\cos a) s^{2} U(s)$ (defined to be positive in the $x$ direction) is given by

$$
Q(s)=2 \pi(1-\cos a) P_{0}\left(\frac{f}{\rho}\right)^{1 / 2}\left(s_{1} s\right)^{5 / 4} \mathrm{e}^{\mathrm{i}\left(z-z_{1}\right)} .
$$

It is interesting to note that for large $z$, the velocity and pressure are predicted to be in phase. Another important characteristic of these expressions is that the variation of $P(s)$ and $U(s)$ no longer depends on $z_{2}$, i.e. the exit boundary (variable $z_{2}$ ) has cancelled out during the derivation of (4.22). The amplitudes of pressure, velocity and flow rate are predicted to vary with rates $s^{-5 / 4}, s^{-3 / 4}$ and $s^{5 / 4}$ respectively. Recall that $s$ decreases as the wave propagates downstream (in the positive $x$ direction), so the pressure and velocity amplitudes increase while the flow rate amplitude decreases.

WKB theory predicts that pressure increases with rate $Y^{-1 / 2}$ and flow rate varies as $Y^{1 / 2}$, where $Y$ is the admittance of the vessel (Lighthill 1975; Pedley 1980; Fung 1996). Using the definition $Y=A / \rho \lambda$ and substituting the expressions for the area $A$ and wave velocity $\lambda$, we find

$$
Y(s)=2 \pi(1-\cos a)\left(\frac{f}{\rho}\right)^{1 / 2} s^{5 / 2},
$$

which shows that pressure and flow rate should vary with rate $s^{-5 / 4}$ and $s^{5 / 4}$, exactly as predicted by (4.22). It can also be seen that we can relate $Q(s)$ and $P(s)$ with

$$
Q(s)=Y(s) P(s) \text {. }
$$

It is also interesting to investigate the time-averaged energy flux. Substituting in (4.12), we find that it is equal to

$$
E(s)=\left|P_{0}\right|^{2} \pi(1-\cos a)\left(\frac{f}{\rho}\right)^{1 / 2} s_{1}^{5 / 2} .
$$

This is identical to (4.20), obtained for very thin rings without reflection. The same conclusion can also be found if we substitute (4.22) into the definition of $w_{1}$ :

$$
w_{1}=\frac{1}{2 \lambda \rho}\left(p+\lambda \rho u_{s}\right)=0,
$$

i.e. there is no reflected wave. The conclusion from this section is that for large values of $z$, the reflected wave $w_{1}$ vanishes and the results naturally yield the well-known WKB solution.

\section{Incorporation of viscous effects}

The theoretical analysis presented so far has ignored the viscous effects. In this section we propose the incorporation of viscous effects on the derived analytic solution through the pulsatile velocity profile of Womersley (1955). This profile is valid for a rigid tube as well as an elastic tube in which there is no longitudinal wall motion (Pedley 1980). The original expression of the profile is written in terms of an oscillating pressure gradient at angular frequency $\omega$, but in this paper we follow the formulation of Reymond et al. (2009) that uses the mean velocity. Using the variables $s, \theta$ we have

$$
u_{s}(s, \theta, t)=\operatorname{Re}\left\{U(s)\left[1-\frac{\mathrm{J}_{0}\left(i^{3 / 2} a_{\omega} \sin \theta / \sin a\right)}{\mathrm{J}_{0}\left(i^{3 / 2} a_{\omega}\right)}\right] \frac{1}{1-F_{10}} \mathrm{e}^{\mathrm{i} \omega t}\right\},
$$


where $F_{10}\left(a_{\omega}\right)$ is the Womersley function given by

$$
F_{10}\left(a_{\omega}\right)=\frac{2 \mathrm{~J}_{1}\left(i^{3 / 2} a_{\omega}\right)}{i^{3 / 2} a_{\omega} \mathrm{J}_{0}\left(i^{3 / 2} a_{\omega}\right)} .
$$

In (5.1) and (5.2), $\mathrm{J}_{0}$ and $\mathrm{J}_{1}$ are the Bessel functions of zeroth and first order respectively, and $a_{\omega}$ is the local Womersley parameter, defined as $a_{\omega}=s \sin a \sqrt{\omega \rho / \mu}$ ( $\mu$ is fluid viscosity). The original derivation assumes a cylindrical tube with constant radius but in the present paper the effect of cross-sectional variation for conical vessels is accounted for by calculating $a_{\omega}$ locally. From (5.1) the shear stress $\tau_{w}$ (positive in the $x$ direction) can be computed as

$$
\tau_{w}=-\operatorname{Re}\left\{U(s) \mu \frac{\mathrm{i}}{2} \frac{a_{\omega}^{2}}{s \sin a} \frac{F_{10}}{1-F_{10}} \mathrm{e}^{\mathrm{i} \omega t}\right\} .
$$

In (5.1) and (5.3), $U(s)$ is the mean flow velocity in the cross-section, i.e. it is given by (4.6). For example, for the specific case examined in $\S 4.3$, the explicit expression for the velocity profile is

$$
u_{s}=\operatorname{Re}\left\{\frac{P_{0}}{i}\left(\frac{f}{\rho} s_{1}\right)^{1 / 2} \frac{C \mathrm{~J}_{4 / 3}(z)+\mathrm{Y}_{4 / 3}(z)}{C \mathrm{~J}_{1 / 3}\left(z_{1}\right)+\mathrm{Y}_{1 / 3}\left(z_{1}\right)}\left[1-\frac{\mathrm{J}_{0}\left(i^{3 / 2} a_{\omega} \sin \theta / \sin a\right)}{\mathrm{J}_{0}\left(i^{3 / 2} a_{\omega}\right)}\right] \frac{\mathrm{e}^{\mathrm{i} \omega t}}{1-F_{10}}\right\} .
$$

The validity of this approximation will be examined in the next section.

\section{Comparison between analytical and numerical results}

In order to confirm the validity of these results and their applicability for modelling the wave propagation in the mammalian cardiovascular system, numerical fluid-structure interaction (FSI) simulations were performed. Details on material properties, vessel dimensions, inlet conditions, mesh sizes and time steps are provided in table 1. In order to check the grid independence of the results, computations were carried out with two meshes.

Based on the data shown in this table, the velocity of propagation obtained from (3.3) varies from 5.77 to $7.15 \mathrm{~m} \mathrm{~s}^{-1}$, at the inlet and outlet of the vessel respectively. The average velocity is $6.46 \mathrm{~m} \mathrm{~s}^{-1}$. This is a physiologically relevant case, in which the wavelength is $\sim 3.2 \mathrm{~m}$, but the vessel is much shorter and the properties change significantly from the inlet to the outlet.

The numerical methodology for the solution of the coupled fluid/structure problem proposed by the author (Papadakis 2008) was used. For the wall, the full elastodynamic set of equations that describe a linear, elastic, Hookean and incompressible material was solved. At the outlet the condition

$$
u_{n}-2 \sqrt{\frac{2}{\rho}}\left(\sqrt{p+\beta \sqrt{A_{\text {out }}}}-\sqrt{\beta \sqrt{A_{\text {out }}}}\right)=0
$$

was applied, where $p$ and $u_{n}$ are the pressure and face-normal velocity at the cell boundaries, $\beta=\left(\sqrt{\pi} h E / 0.75 A_{\text {out }}\right), h$ is the thickness of the wall and $A_{\text {out }}=\pi r_{\text {out }}^{2}$ is the outlet area. This expression describes mathematically the condition that there is no incoming (incident) wave from the outlet of the domain. The linearized form of (6.1) corresponds to the boundary condition given by $(4.15 b)$. It was decided to keep the nonlinear expression in agreement with the fact that the full nonlinear problem is 
Wall properties

\begin{tabular}{|c|c|}
\hline Modulus of elasticity $(\mathrm{Pa})$ & $10^{6}$ \\
\hline Poisson ratio $(-)$ & 0.5 \\
\hline Density $\left(\mathrm{kg} \mathrm{m}^{-3}\right)$ & 1000 \\
\hline 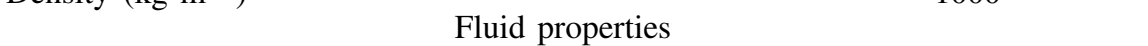 & 0.004 \\
\hline Density $\left(\mathrm{kg} \mathrm{m}^{-3}\right)$ & 1000 \\
\hline \multicolumn{2}{|c|}{ Basic dimensions of vessel } \\
\hline Outlet radius (mm) & 6.5 \\
\hline Wall thickness (mm) & 0.5 \\
\hline Length (mm) & 100 \\
\hline Cone angle $\left({ }^{\circ}\right)$ & 2 \\
\hline \multicolumn{2}{|c|}{ Conditions at inlet } \\
\hline Pressure variation with time $(\mathrm{Pa})$ & $p_{0} \sin (2 \pi f t)$ \\
\hline Frequency, $f$, of inlet pressure $(\mathrm{Hz})$ & 2 \\
\hline Amplitude, $p_{0}$, of inlet pressure $(\mathrm{Pa})$ & 1000 \\
\hline Mesh $n x \times\left(n r_{f}+n r_{w}\right)^{*} /$ time step (s) & $\begin{array}{c}60 \times(19+5) / 10^{-4} \\
120 \times(39+10) / 5 \times 10^{-5}\end{array}$ \\
\hline
\end{tabular}

TABLE 1. Properties of wall and fluid, geometric and numerical parameters. ${ }^{*} n x$ denotes the number of cells in the $x$ direction and $n r_{f}$ and $n r_{w}$ the number of cells in the $r$ direction for the fluid and wall respectively.

solved. More details about the derivation of this expression and its implementation into a finite-volume pressure-correction solver are given in Papadakis (2009). In order to compare with the analytical solution presented in the previous sections, the computational fluid dynamics results are averaged in a cross-section. It must be stressed that simulations with flow reversals at the exit of a domain are not trivial and can create numerical instabilities. The numerical methodology and boundary condition used in this study proved to be very robust for such flow conditions and no numerical problems were encountered.

Figure 2 shows the variation of area-averaged pressure and velocity at the middle of the vessel $(x=L / 2)$ against time for one period. Results are presented for both meshes and it can be clearly seen that they are almost identical. The matching with the analytic solution is very good; the relative error for the peak values is less than $1 \%$ for pressure and $1.6 \%$ for velocity. There is a small deviation close to $t=0$, which is due to the initial conditions used in the numerical simulations (all variables were set equal to 0 ). The fact that the peaks are predicted correctly shows that the wave propagation velocity is also predicted well.

The variation of the wave energy at the same location is also shown in figure 2 (results only with the fine mesh are shown). The period of oscillation is half that of external pressure (4.11). The good matching with the analytic solution (relative error $1.2 \%$ at the peak) indicates that at this low frequency the effect of viscosity is small. The results are slightly non-symmetric as can be seen from the peak values (slightly larger or smaller than the analytic solution). This is probably due to the fact that at these time instants, which correspond to large axial velocities, the effect of nonlinearity is more pronounced, and this leads to the loss of symmetry.

Figure 3 shows the variation of pressure and velocity against distance along the vessel for eight time instants in one period. Again there is very good agreement 
New analytic solutions for wave propagation in flexible, tapered vessels

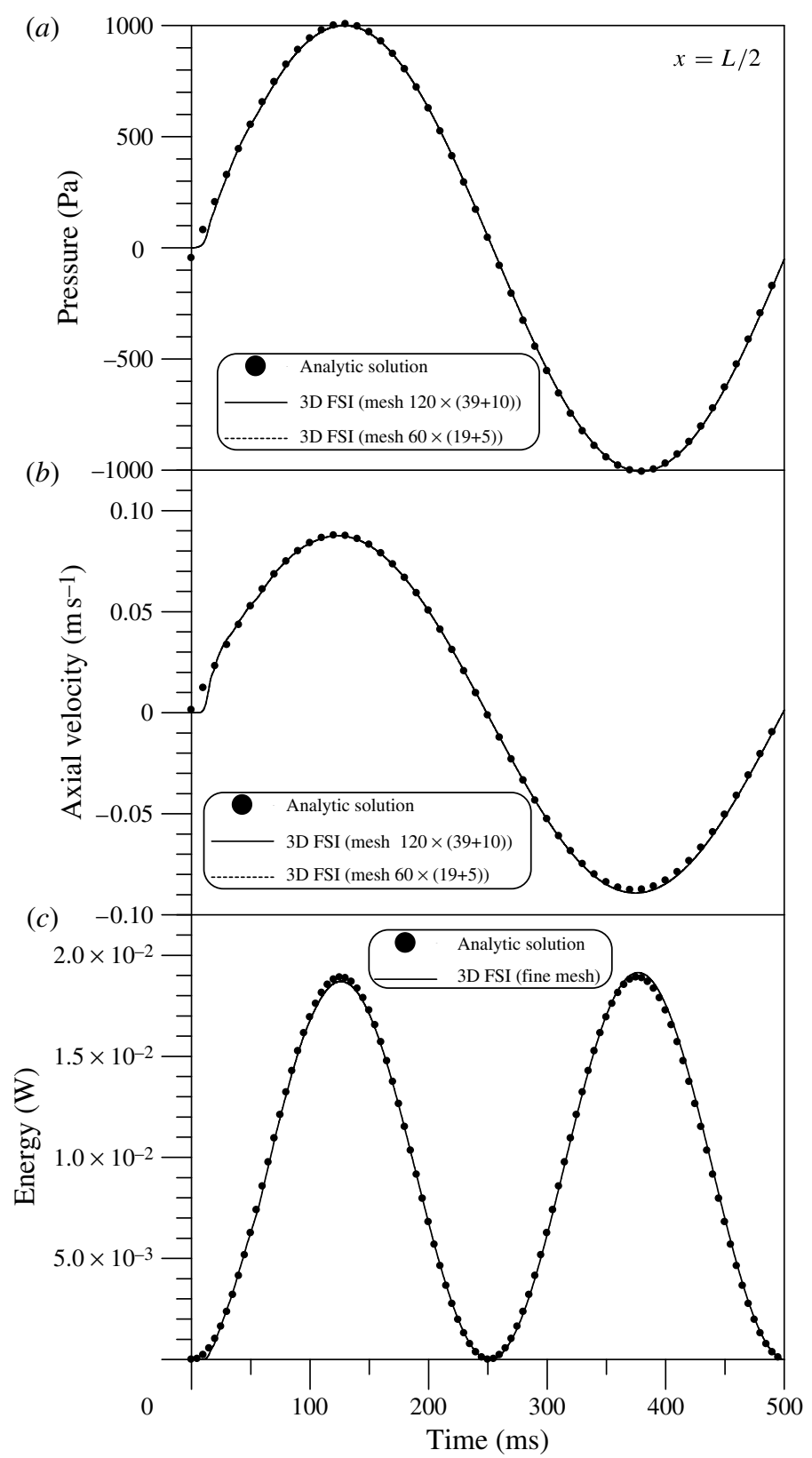

FIGURE 2. Variation of (a) pressure, $(b)$ velocity and $(c)$ energy for one period at $x=L / 2$.

between the numerical predictions and the analytic solution derived in $\S 4.3$. Note that the variation of pressure along the vessel is not strong, which is expected because of the low frequency of the external pressure wave. It can be easily computed that the wave propagates from one end of the vessel to the other in $\sim 0.03 T$, during which the variation of pressure at the inlet is small. On the other hand, the variation of axial velocity (induced by the pressure waveform) along $x$ is much stronger. It can also be seen that the velocity and pressure are not in phase. For example, at $x=0$ and at time 

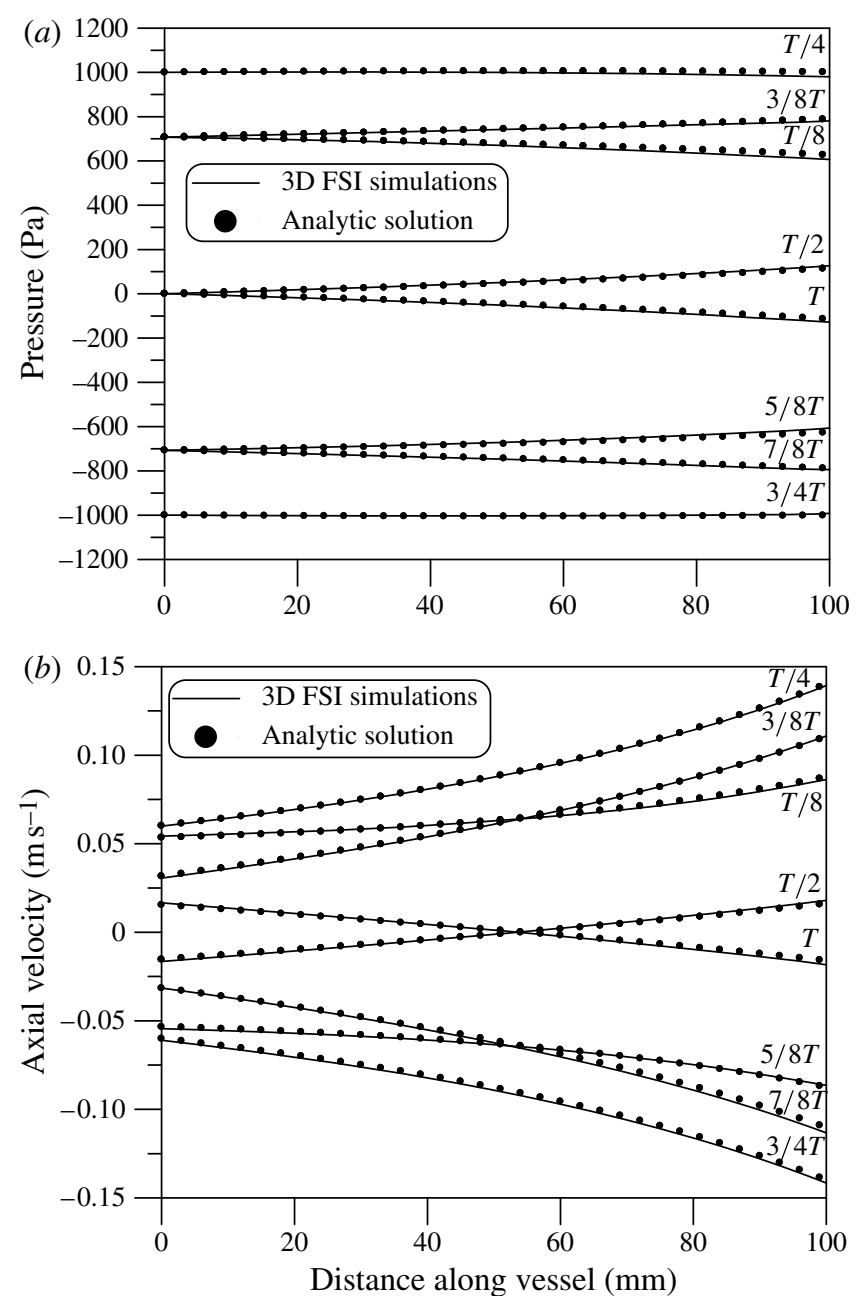

FIGURE 3. Variations of $(a)$ pressure and $(b)$ axial velocity along the distance of the vessel at several time instants.

instants $t=T / 2$ and $T$ at which pressure is equal to 0 , velocity has a finite, non-zero, value. The reason for this is that the axial velocity at the inlet is affected by the continuous reflection of the forward-propagating wave due to the change in the vessel diameter. Without reflection, velocity and pressure are in phase, as in the case of wave propagation within a cylindrical vessel. This phase difference cannot be captured using WKB theory as already explained earlier. It is also interesting to note that at two time instants, $T / 2$ and $T$, the velocity changes sign from one end of the vessel to the other. This, of course, would have violated the continuity equation for a rigid wall, but is clearly the correct solution for elastic, deformable walls.

In order to further examine the deviation of the results from rigid walls, figure 4 shows the axial velocity at three time instants obtained from the continuity equation $(\partial / \partial s)\left(s^{2} \bar{u}_{s}\right)=0$ using the same velocity at $x=0$ (the value shown in figure $\left.3 b\right)$. It can be clearly seen that the effect of tube deformation is important and increases with distance. 
New analytic solutions for wave propagation in flexible, tapered vessels

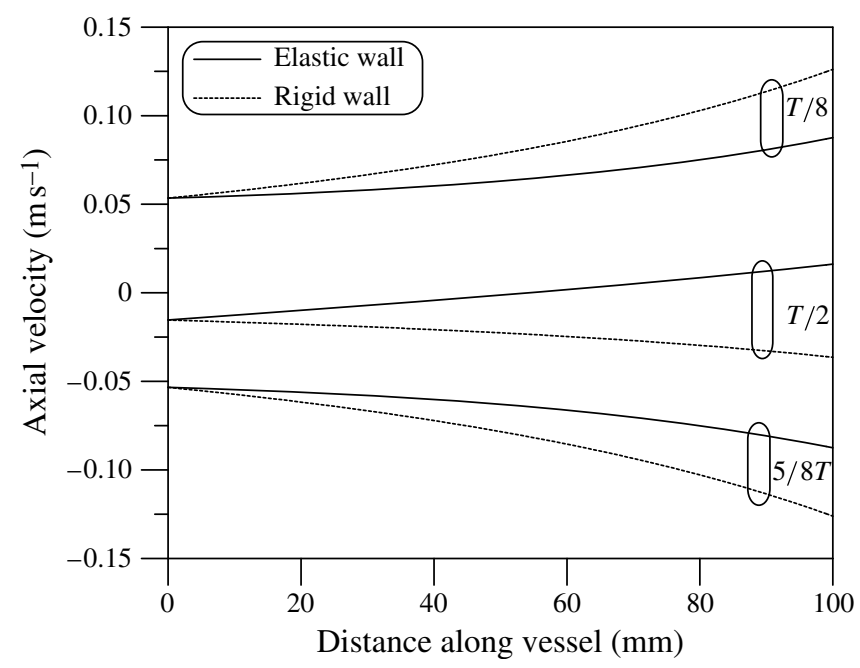

FIGURE 4. Comparison of axial velocity variations for elastic and rigid walls (same velocity at the inlet).

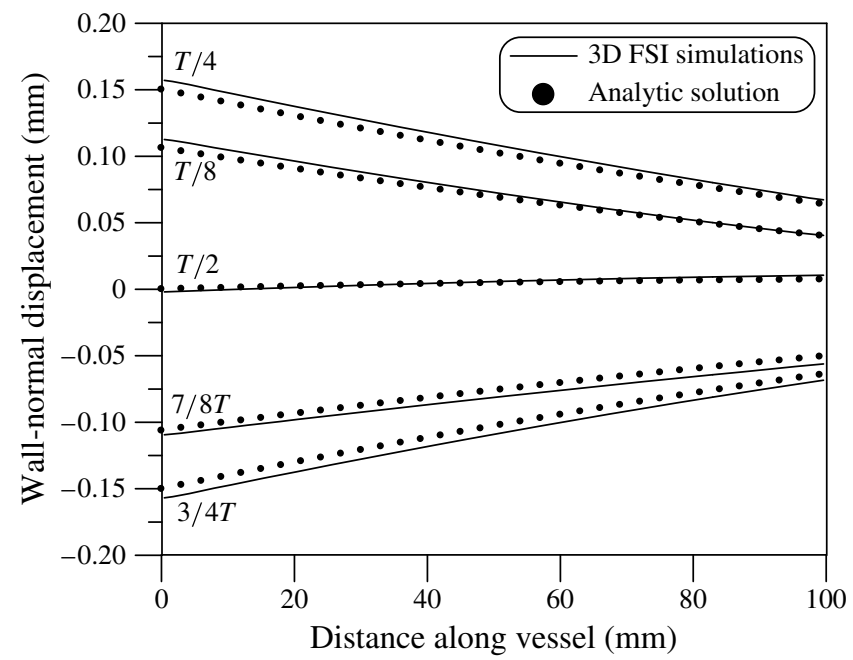

FIGURE 5. Variation of wall-normal displacement along the distance of the vessel at five time instants.

Figure 5 compares the analytical and numerical results for the wall-normal displacement at five time instants in one period. For the analytic solution, (A 8) was employed, using the instantaneous cross-sectional pressure. Recall that in the numerical solution the full set of equations of motion of a deforming elastic material was solved, and no assumption was made with respect to the thickness of the wall. It can be seen that the matching between the two solutions is good and the differences are small $(\sim 4.6 \%$ at $t=T / 4)$. This difference is most probably attributed to the assumptions used in deriving wall model (A 8) (thin and short conical ring, negligible longitudinal displacement). 

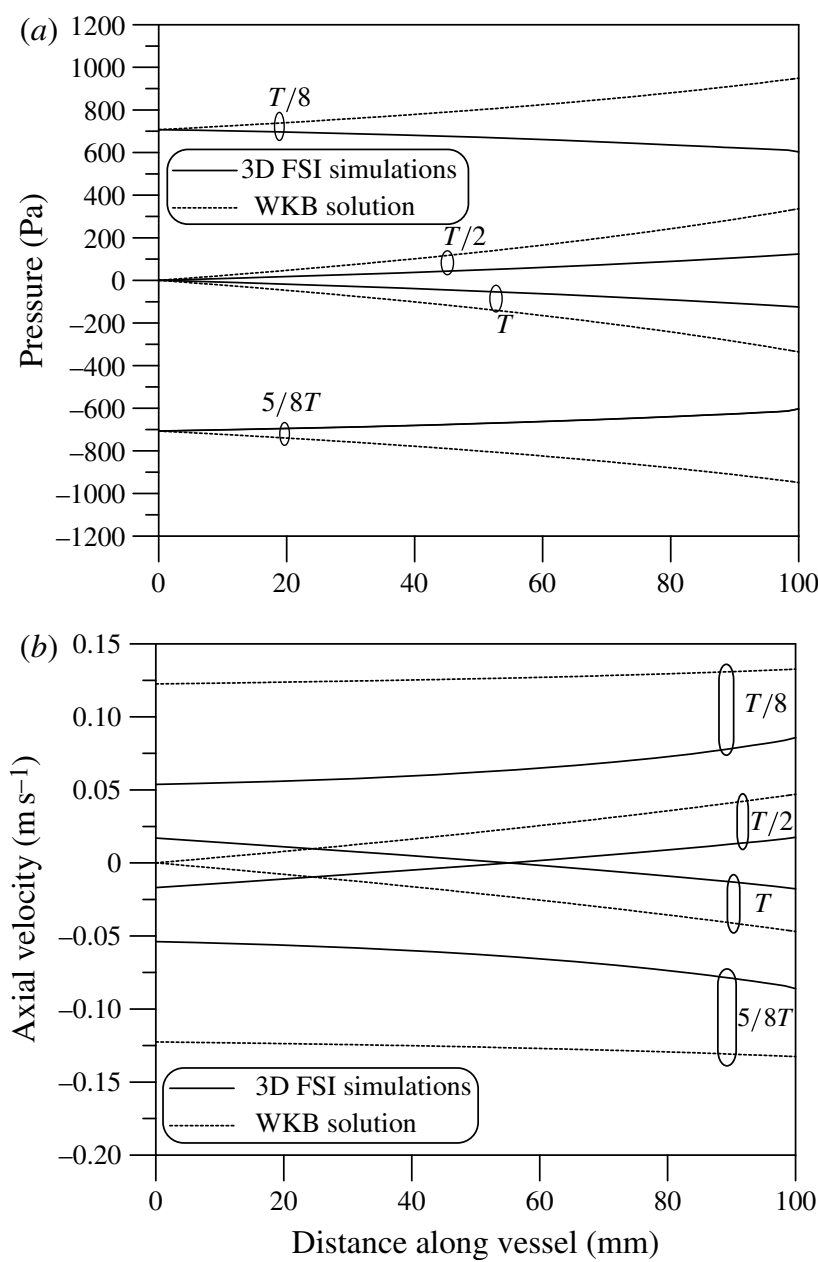

FIGURE 6. Comparison of $(a)$ pressure and $(b)$ velocity between numerical results and WKB theory.

Figure 6 compares the variation of velocity and pressure with the predictions of WKB theory, (4.22). In order to avoiding cluttering up the figure, results for only four time instants are shown. The WKB results clearly do not match the numerical results and the difference is quite substantial. For pressure this difference increases downstream, with WKB theory predicting stronger variation with distance. Axial velocities seem to be displaced in the vertical axis, most evident at $t=T / 2$ and $T$. This is due to the wrong phase of the velocity as mentioned earlier.

The time-averaged energy flux of the wave remains constant along the length of the tube, as demonstrated by (4.14). However this value depends on the length of the tube $L$ ( $L$ is incorporated within the argument $z_{2}$ of coefficient $C$ ). Figure 7 shows the variation of the time-averaged energy flux with the length of the tube, $L$. Note the change in the label of the horizontal axis with respect to the previous figures (length of vessel, instead of distance along the vessel). WKB solution predicts that this value is independent of $L$. However, the present analytic solution indicates that for low frequencies, increasing the length of the tube reduces the time-averaged energy of the wave. This can be explained if we examine $(4.10 b)$, which expresses the energy with 


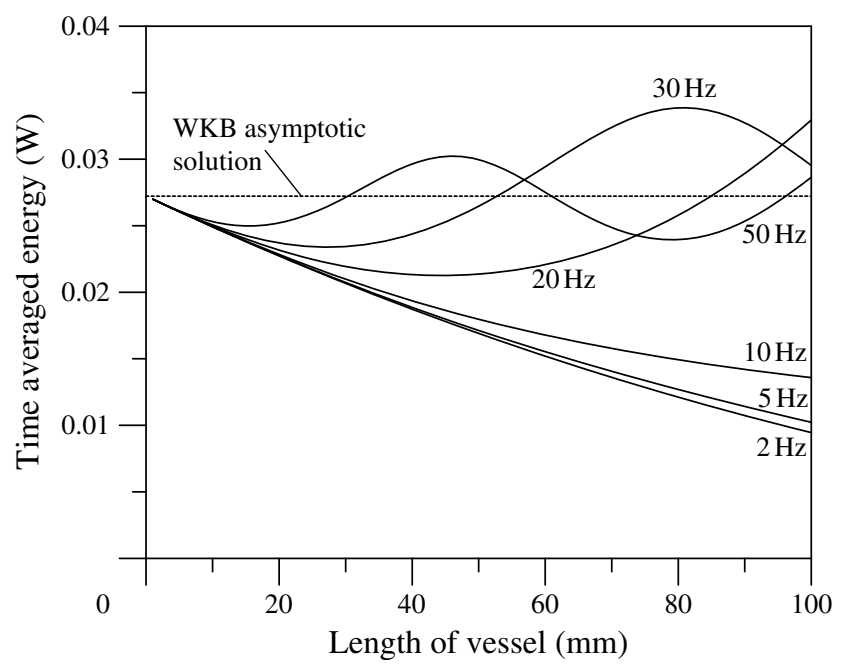

FIGURE 7. Variation of time-averaged energy with the length of the tube.

respect to the characteristic variables, $w_{1}, w_{2}$. The presence of $w_{1}$ waves generated due to reflection from the tapered wall reduces the energy. When the length of the tube is null (i.e. we have a thin ring), there is no reflection and the result matches WKB theory as already discovered in $\S 4.4$. As the length increases, the reflected waves $w_{1}$ become stronger and this leads to reduction of energy, as per $(4.10 b)$. However, when the frequency increases, an interesting phenomenon occurs. The energy levels actually increase and can exceed the value obtained without reflection. A likely explanation is as follows. The $w_{1}$ wave propagates upstream, but is reflected at the entrance, to satisfy the entrance condition $(4.15 a)$. This leads to a stronger $w_{2}$ wave and thus larger energy.

In order to further investigate this phenomenon, in figure 8 the time-averaged energy for a vessel with $L=0.1 \mathrm{~m}$ is plotted against the frequency of the external pressure wave. It can be seen that for particular frequencies, some of which are denoted by black dots in the figure, local maxima and minima exist. The figure also proves that when frequency increases, which means that argument $z$ increases, the solution tends to the WKB result. It can be seen that the exact solution oscillates about this asymptotic value.

The numerical solutions allow us to study in more detail the flow and pressure fields. Figure 9 shows the contour plot of pressure at two time instants, $t=T / 2$ and $T$, at which the inlet pressure is 0 . The pressure distribution is approximately constant in the cross-section of the vessel, and the pressure field is smooth, even close to the exit. These characteristics are the same for all the time instants examined.

On the other hand, velocities have stronger variation across the cross-section. Figure 10 shows radial profiles of axial velocity predicted by the numerical methodology and the approximate expression (5.4) at $x=L / 2$. The local Womersley parameter $a_{\omega}$ is equal to 14.6. The matching between the two solutions is good, especially close to the wall, at all time instants. The maximum differences $(\sim 5-6 \%)$ are localized at those radial locations where the velocities are maximized (time instants $T / 4$ and $6 T / 8$ ), most probably due to the presence of nonlinear effects. The radii of the peaks are correctly predicted. It is clear that the velocities at different radii are not in phase (this is shown by the crossing of the profiles, for example at $5 T / 8$ and $7 T / 8$ ). 


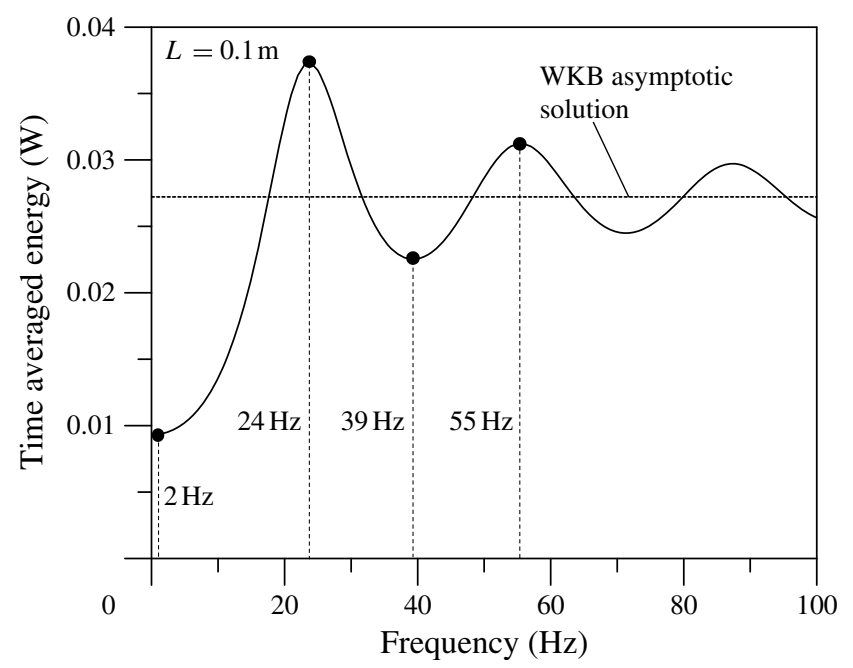

FIGURE 8. Variation of time-averaged wave energy flux with frequency.

Predictions of similar quality were obtained for other axial locations, from the inlet and up to $\sim 80 \%$ of the length of the vessel. Closer to the exit the two solutions deviate because the boundary condition (6.1) does not account for viscous effects and leads to profile distortions. The fact that there is good matching between the two solutions close to the wall (at least away from the exit) is important, because it means that the wall shear stress can be estimated with good accuracy from (5.3). The proposed method for inclusion of viscous effects therefore works for the case examined, making the analytic solution useful for estimating the variation of shear stress along conical vessels.

The proposed model can be extended to include the effects of visco-elasticity, steady velocity component and variable wall thickness. This will allow, for the first time, the theoretical analysis of continuous wave reflection in more realistic stenotic flows, for example due to atherosclerosis. It is expected that such an analysis will provide new insights into cardiovascular physiology and will help with the interpretation of observed pulse waveforms.

\section{Conclusions}

To the best of our knowledge, this is the first time that closed-form analytic solutions for the pressure distribution and axial velocity for waves propagating inside a conically tapered tube have been presented. The derived solutions are valid for the range of parameters encountered in physiological flow conditions in mammalian cardiovascular systems. The results naturally approach the WKB solution for the range of parameters for which this approximate solution is valid. The continuous variation of the cross-sectional area leads to continuous reflection of the propagating waves in the positive and negative axial directions. The two waves are intimately coupled, they propagate together, and cannot be treated independently. Viscous effects are incorporated by coupling the derived analytic solution with the Womersley velocity profile in the radial direction. The results are compared against fluid-structure interaction simulations and good matching is observed. 
New analytic solutions for wave propagation in flexible, tapered vessels

(a)

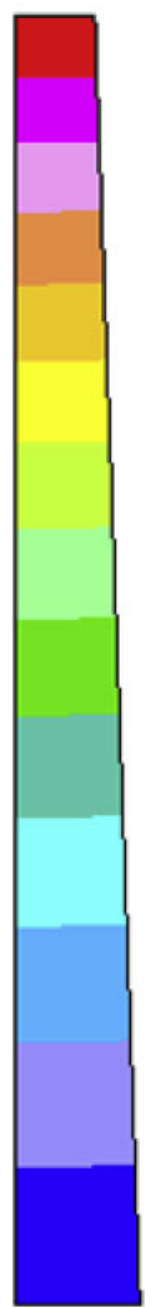

(b)

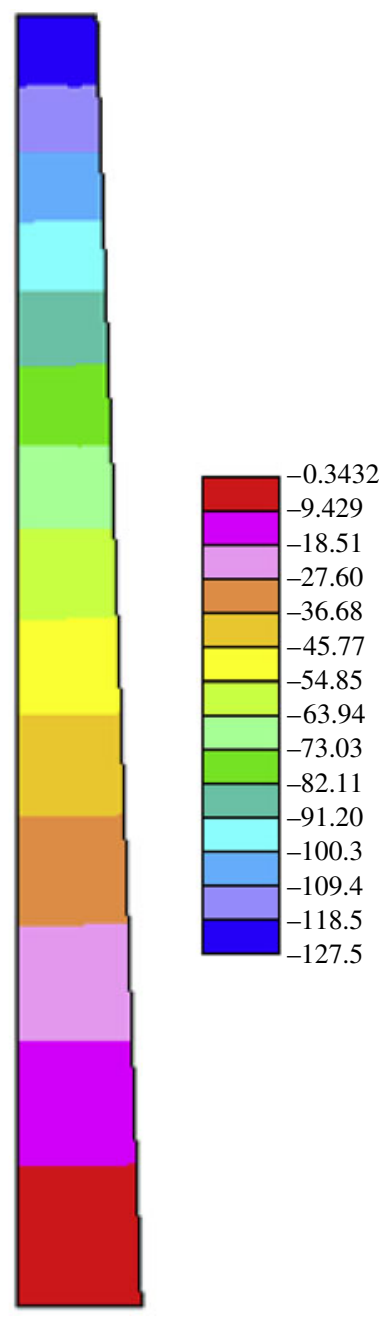

FIGURE 9. (Colour online available at journals.cambridge.org/flm) Contour plots of pressure (in Pa) at $t=T / 2(a)$ and $t=T(b)$.

\section{Appendix. Variation of wall-normal displacement with internal pressure for conical rings}

Let us assume a conical ring filled with liquid with internal pressure $p$ (see figure 11). In this appendix, an expression will be derived that links the wall-normal displacement, $\eta$, with $p$.

It is assumed that the ring is located at distance $s$ from the origin of the coordinate system $O$ and has very small longitudinal length. The membrane stress resultant in the circumferential $(\varphi)$ direction assuming negligible longitudinal displacement (i.e. plane strain conditions) is (Flügge 1960)

$$
N_{\varphi}=D\left(\frac{1}{s} \frac{\eta}{\tan a}\right)
$$




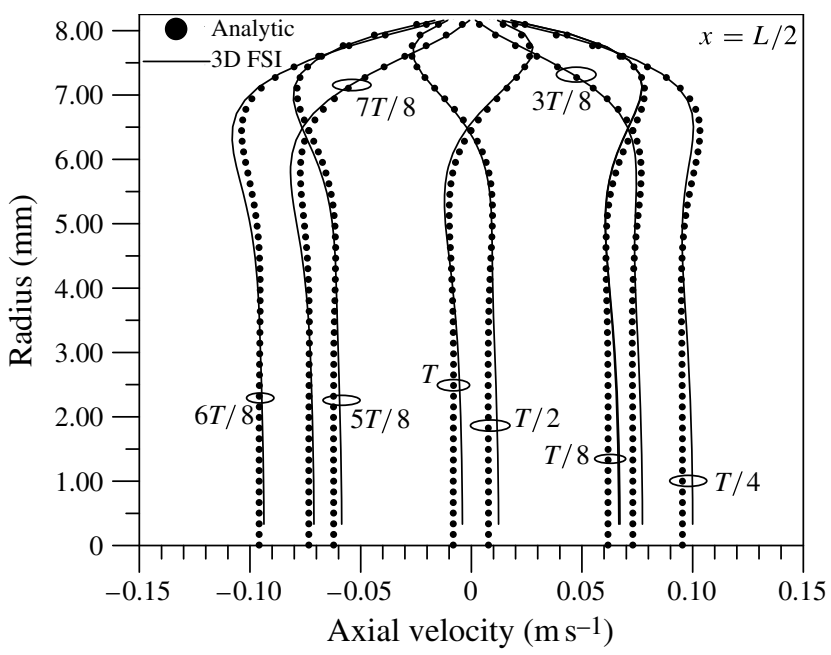

FIGURE 10. Comparison between numerical results and analytic solution for radial velocity profiles at several time instants.

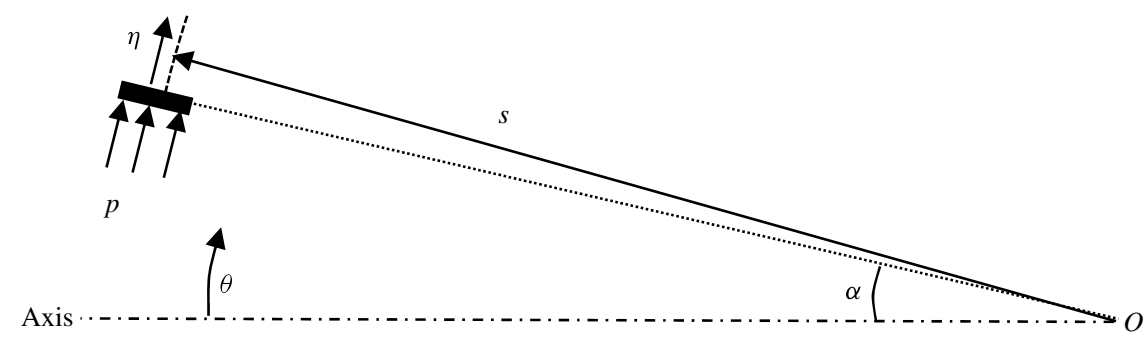

FIGURE 11. A thin conical ring.

where $\eta$ is the deformation in the $\theta$ direction (as shown in figure 11) and $D$ is the extensional rigidity of the wall given by

$$
D=\frac{E h}{1-v^{2}},
$$

where $h$ is the thickness of the wall, $E$ the modulus of elasticity and $v$ the Poisson ratio. The equation of motion in the wall-normal direction is (Flügge 1960)

$$
-\frac{N_{\varphi}}{s \tan a}+p=h \rho_{w} \frac{\partial^{2} \eta}{\partial t^{2}},
$$

where $\rho_{w}$ is the density of the wall. Since the characteristic frequency is small, the inertia terms can be ignored, and substituting the stress definition (A 1) into (A 3) we get

$$
-\frac{D}{s \tan a}\left(\frac{1}{s} \frac{\eta}{\tan a}\right)+p=0 .
$$


This equation can be solved directly for $\eta$ :

$$
\eta=p \frac{\left(1-v^{2}\right) \tan ^{2} a}{E h} s^{2} .
$$

In terms of the local radius $r=s \sin a$, the expression for $\eta$ can be written as

$$
\eta=p \frac{\left(1-v^{2}\right)}{E h \cos ^{2} a} r^{2}
$$

In the limiting case for a circular cylinder with radius $R$, i.e. when the angle $a=0$, (A 6) reduces to

$$
\eta_{a=0}=\frac{p R^{2}}{E h}\left(1-v^{2}\right)
$$

which is the well-known expression for radial displacement under plane strain conditions (no longitudinal displacement).

For incompressible material with Poisson ratio $v=0.5$, (A 5) and (A 6) are simplified to

$$
\eta=p \frac{0.75 \tan ^{2} a}{E h} s^{2}=p \frac{0.75}{E h \cos ^{2} a} r^{2}
$$

\section{REFERENCES}

Alastruey, J., Khir, A. W., Matthys, K. S., Segers, P., Sherwin, S. J., Verdonck, P. R., PARKer, K. H. \& Peiró, J. 2011 Pulse wave propagation in a model human arterial network: assessment of 1D visco-elastic simulations against in vitro measurements. J. Biomech. 44, 2250-2258.

ATABEK, H. B. 1968 Wave propagation through a viscous fluid contained in a tethered, initially stressed, orthotropic elastic tube. Biophys. J. 8, 626-649.

ATABEK, H. B. \& LEW, H. S. 1961 Wave propagation through a viscous incompressible fluid contained in an initially stressed elastic tube. Biophys. J. 6, 481-503.

Bender, C. M. \& Orszag, S. A. 1999 Advanced Mathematical Methods for Scientists and Engineers: Asymptotic Methods and Perturbation Theory. Springer.

Bessems, D., Giannopapa, C. G., Rutten, M. C. M. \& Van De Vosse, F. N. 2008 Experimental validation of a time-domain-based wave propagation model of blood flow in viscoelastic vessels. J. Biomech. 41, 284-291.

Bessems, D., RutTen, M. \& VAn DE Vosse, F. 2007 A wave propagation model for blood flow in large vessels using an approximate velocity profile function. J. Fluid Mech. 580, 145-168.

Cox, R. H. 1969 Comparison of linearized wave propagation models for arterial blood flow analysis. J. Biomech. 2, 251-265.

Einav, S, Aharoni, S. \& Manoach, M. 1998 Exponentially tapered transmission line model of the arterial system. IEEE Trans. Biomed. Engng 35 (5), 333-339.

FLÜGGE, W. 1960 Stresses in Shells. Springer.

FUnG, Y. C. 1996 Biomechanics: Circulation, 2nd edn. Springer.

Holmes, M. H. 1998 Introduction to Perturbation Methods, Texts in Applied Mathematics, vol. 20. Springer.

Hughes, T. J. R \& Lubliner, J. 1973 On the one-dimensional theory of blood flow in the large vessels. Math. Biosci. 18, 161-170.

Korteweg, D. J. 1878 Über die Fortpflanzungsgeschwindigkeit des Schalles in elastischen Röhren. Ann. Phys. Chem. (NS) 5, 525-527.

LeVeque, R. J. 2002 Finite Volume Methods for Hyperbolic Problems. Cambridge University Press. 
Lighthill, J. 1975 Pulse propagation theory. In Mathematical Biofluid Dynamics, CBMS-NSF Regional Conference Series in Applied Mathematics, vol. 17. SIAM.

Matthys, K. S., Alastruey, J., Peiro, J., Khir, A. W., Segers, P., Verdonck, P. R., PARKER, K. H. \& SHERWIN, S. J. 2007 Pulse wave propagation in a model human arterial network: assessment of 1D numerical simulations against in vitro measurements. J. Biomech. 40, 3476-3486.

Moens, A. I. 1877 Over de Voortplantingssnelheid van den Pols. Van Doesburgh.

Myers, L. J. \& CAPPER, W. L. 2004 Exponential taper in arteries: an exact solution of its effect on blood flow velocity waveforms and impedance. Med. Engng Phys. 26, 147-155.

PAPADAKIS, G. 2008 A novel pressure-velocity formulation and solution method for fluid-structureinteraction problems. J. Comput. Phys. 227, 3383-3404.

PAPADAKIS, G. 2009 Coupling 3D and 1D fluid-structure-interaction models for wave propagation in flexible vessels using a finite volume pressure-correction scheme. Commun. Numer. Meth. Engng 25, 533-551.

Pedley, T. J. 1980 The Fluid Mechanics of Large Blood Vessels. Cambridge University Press.

Pedley, T. J. 2000 Blood flow in arteries and veins. In Perspectives in Fluid Dynamics (ed. G. K. Batchelor, H. K. Moffatt \& M. G. Worster), pp. 105-158. Cambridge University Press.

Pedley, T. J. 2003 Mathematical modelling of arterial fluid dynamics. J. Engng Math. 47, 419-444.

Press, W. H., Teukolsky, S. A., Vetterling, W. T. \& Flannery, B. P. 1997 Numerical recipes in Fortran 77. In The Art of Scientific Computing, 2nd edn. Cambridge University Press.

RÅde, L. \& Westergren, B. 1999 Mathematics Handbook for Science and Engineering, 4th edn. Springer.

Reymond, P., Merenda, F., Perren, F., Rufenacht, D. \& Stergiopoulos, N. 2009 Validation of a one-dimensional model of the systemic arterial tree. Am. J. Physiol. Heart Circ. Physiol. 297, H208-H222.

Sherwin, S. J., Franke, V., Peiró, J. \& Parker, K. 2003 One-dimensional modelling of a vascular network in space-time variables. J. Engng Math. 47, 217-250.

Steele, B. N., Olufsen, M. S. \& TAYlor, C. A. 2007 Fractal network model for simulating abdominal and lower extremity blood flow during resting and exercise conditions. Comput. Meth. Biomech. Biomed. Engng 10 (1), 39-51.

Steele, B. N., Valdez-Jasso, D., Haider, M. A. \& Olufsen, M. S. 2011 Predicting arterial flow and pressure dynamics using a 1D fluid dynamics model with a visco-elastic wall. SIAM J. Appl. Math. 71 (4), 1123-1143.

VAn de Vosse, F. N. \& Stergiopoulos, N. 2011 Pulse wave propagation in the arterial tree. Annu. Rev. Fluid Mech. 43, 467-499.

WARSI, Z. U. A. 1999 Fluid Dynamics: Theoretical and Computational Approaches, 2nd edn. CRC Press.

WITZIG, K. 1914 Über erzwungene Wellenbewegungen zäher, inkompressibler Flüssigkeiten in elastischen Röhren. Inaugural Dissertation, University of Bern.

Womersley, J. R. 1955 Oscillatory motion of a viscous liquid in a thin-walled elastic tube. Part I. The linear approximation for long waves. Phil. Mag. 46 (7), 199-219.

Womersley, J. R. 1957 Oscillatory flow in arteries: the constrained tube as a model of arterial flow and pulse transmission. Phys. Med. Biol. 2, 178-187. 